\title{
Short communication: Performance of a blocking antibody ELISA bulk-tank milk test for detection of dairy sheep flocks exposed to border disease virus
}

\author{
F. Corbière, ${ }^{*}{ }^{1}$ C. Pouget, $\neq$ E. Bernardin, ${ }^{*}$ R. Brugidou, $\S$ and F. Schelcher ${ }^{\star} \dagger$ \\ *Université de Toulouse, Ecole Nationale Vétérinaire, 23 chemin des Capelles, F-31076 Toulouse Cedex 3, France \\ †UMT Santé des Troupeaux de Petits Ruminants, UMR 1225, 23 chemin des Capelles, F-31076 Toulouse Cedex 3, France \\ †Fédération des organismes de défense sanitaire de l'Aveyron, Avenue des Ebénistes, F-12032 Toulouse cedex, France \\ $\S$ Aveyron Labo, Rue des Artisans, F-12301 Rodez cedex 9, France
}

\begin{abstract}
The aim of this study was to investigate the test characteristics of a blocking antibody ELISA applied to bulk-tank milk (BTM) samples for the detection of dairy sheep flocks positive for antibodies to border disease virus. In 161 flocks recruited in 2009 and 2010, the antibody inhibition percentage (AIP) in BTM was compared with the prevalence estimate of antibodypositive ewes obtained from an age-representative sample of 45 milking ewes. A strong negative exponential relationship $\left(\mathrm{R}^{2}=0.89\right)$ was found between AIP in BTM and seroprevalence level. Using receiver operating characteristic analysis, the best AIP decision threshold in BTM to discriminate between low $(<10 \%)$ and high $(\geq 10 \%)$ antibody-positive flocks was $65 \%$. Diagnostic performance estimates based on observed seroprevalence levels and Monte Carlo simulations showed that this threshold value was associated with high sensitivity and specificity $(91.9 \pm 5.5 \%$ and $95.9 \pm$ $1.6 \%$, respectively), whereas the $80 \%$ decision threshold recommended in dairy cows yielded lower specificity $(83.6 \pm 2.0 \%)$. Results obtained from the same flocks during 2 subsequent milking campaigns showed that the $65 \%$ AIP cut-off value was associated with fewer false-positive results and is preferred. Testing of BTM samples could be a powerful tool in inferring border disease virus seroprevalence in a flock and in Pestivirus control schemes in dairy sheep flocks.
\end{abstract}

Key words: dairy sheep, border disease, bulk-tank milk, test characteristic

\section{Short Communication}

Border disease virus (BDV) is a worldwide-distributed Pestivirus of sheep, closely related to bovine viral diarrhea virus and classical swine fever virus (Valdazo-

Received April 2, 2012.

Accepted July 13, 2012.

${ }^{1}$ Corresponding author: f.corbiere@envt.fr
González et al., 2007; Dubois et al., 2008). Abortion, stillbirths, and unviable lambs caused by BDV are of great concern for sheep producers (Garcia-Pérez et al., 2010). On a larger scale, persistently infected lambs represent a serious threat when sold to fattening units or to other producers as replacement ewes because they efficiently spread the disease (Nettleton et al., 1998). In dairy cattle herds, the analysis of bulk-tank milk (BTM) samples for the presence of antibodies against bovine viral diarrhea virus has been shown to have great epidemiological relevance and is currently part of control schemes in several European countries (Beaudeau et al., 2005; Houe et al., 2006; Lindberg et al., 2006). The use of BTM sample analysis has been recently reported in dairy sheep flocks (Berriatua et al., 2006; Garcia-Pérez et al., 2010). However, the interpretation rules of antibody inhibition percentage (AIP) results in BTM were those recommended for dairy cattle herds and they need to be properly evaluated for application in sheep. Furthermore, the diagnostic performance of antibody measurement in BTM samples has been investigated in dairy cattle (Valle et al., 2001) but is currently unknown in sheep. The aims of our study were, therefore, to investigate the diagnostic performance of a blocking ELISA applied to BTM samples for the detection of dairy sheep flocks positive for BDV antibodies and to provide sheep-specific interpretation rules, if needed.

In total, 184 dairy sheep flocks were recruited between 2009 and 2010 on a voluntary basis. Flock size varied between 100 and 900 adult lactating ewes, with a median flock size of 330 . In 6 surveyed flocks, adults or yearling ewes had been vaccinated using a live attenuated vaccine (Mucosiffa, Merial, Lyon, France) before the study period. Because this type of vaccine induces a strong and persistent anti-BDV p125/p80 antibody response, these flocks were excluded from the analysis. Seventeen other flocks were discarded because the animals from which blood samples were collected were not representative of the age distribution of lactating ewes (only 1- to 2-yr-old ewes). Therefore, 161 flocks were included in the analysis. 
In each flock, blood samples were collected from an age-representative sample of 45 adult lactating ewes. A sample of BTM was collected within 1 to 2 mo and stored at $-20^{\circ} \mathrm{C}$ until analysis. Serum and milk samples were tested for the presence of anti-BDV p125/p80 antibodies by means of a commercial blocking ELISA test (ELISA BVD-MD-BD P80 antibody screening; Idexx, Montpellier, France). At the individual animal level, the manufacturer's guidelines were strictly followed: serum samples with an AIP value $\geq 50 \%$, between 40 and $50 \%$, and $<40 \%$ were considered negative, weakly positive, and positive for BDV antibodies, respectively.

The observed within-flock antibody prevalence (Prev) was calculated as the ratio of seropositive animals to the number of sampled animals in each flock. The relationship between BDV seroprevalence in each flock and BTM AIP values were evaluated using general linear regression models after adjusting for the number of milking ewes and the number of milkings contributing to the bulk tank.

The AIP cut-off values for the blocking ELISA applied to BTM samples were determined by means of receiver operating characteristic analysis to achieve the best relationship between test sensitivity $(\mathbf{S e})$ and specificity (Sp). Cut-off values were selected based on the maximization of Youden's index $(\mathrm{J}=\mathrm{Se}+\mathrm{Sp}-1)$ and efficiency (i.e., proportion of flocks correctly classified by the test applied to BTM). Statistical uncertainty was assessed by calculating exact $95 \%$ binomial confidence intervals $(95 \% \mathrm{CI})$. Bias-corrected and accelerated $95 \%$ CI based on 1,000 bootstrap replications were computed for Youden's index (Schisterman and Perkins, 2007).

The test characteristics were defined as the ability of the blocking ELISA applied to BTM samples to distinguish low-seroprevalence flocks (i.e., prevalence $<10 \%$ ) from all others (i.e., prevalence $\geq 10 \%$ ). In a first approach, Prev was used as a perfect gold standard, so that flocks were classified as negative whenever Prev $<10 \%$ and as positive otherwise. However, because of the small number of sampled animals, Prev may not be considered as a perfect gold standard, because the $10 \%$ cut-off value may be included in its exact binomial $95 \%$ CI. This is the case when 1 (95\% CI: $0.06-11.77 \%)$ to 9 (95\% CI: $9.58-34.60 \%)$ of 45 ewes are antibody positive. To take account of this statistical uncertainty and to better evaluate the BTM test characteristics, Monte Carlo simulations were conducted based on 10,000 iterations. For each flock within each iteration, a seroprevalence value was generated under a $\beta(a, b)$ distribution, where $a=$ number of seropositive animals +1 , and $b=$ number of tested animals - number of seropositive animals +1 .
This distribution closely reflects the probability density function of expected values under a binomial likelihood (Messam et al., 2008). For each flock, a positive or negative status was defined based on its simulated seroprevalence level using the $10 \%$ cut-off value. The test characteristics were computed for each iteration for several AIP decision thresholds. Finally, the overall Se, Sp, and efficiency estimates and 95\% CI were calculated using the median value and the $2.5 \%$ and $97.5 \%$ percentiles of the estimate distributions over the 10,000 iterations.

In 101 flocks (63\%), no animals had detectable level of antibodies. Therefore, in these flocks, under the assumption of an almost-perfect test at the individual animal level and according to the binomial distribution, the upper $95 \%$ confidence limit of the within-flock prevalence of BDV-positive animals was $7.87 \%$.

Results from general linear regression models showed that year of sampling $(P=0.59)$, number of milking ewes $(P=0.41)$, and number of milkings $(P=0.75)$ contributing to the BTM sample had no significant effect of AIP values in BTM. In Figure 1, the observed within-flock seroprevalence estimates (Prev) are plotted against AIP values in BTM. Flocks with prevalence $>60 \%$ had, with 1 exception, AIP in BTM of $<30 \%$. Conversely, AIP in BTM $>65 \%$ was associated with within-flock prevalence of $<10 \%$ in all but 1 flock.

The best-fit regression model to estimate Prev was a negative exponential relationship $\left(R^{2}=0.89\right)$, as follows:

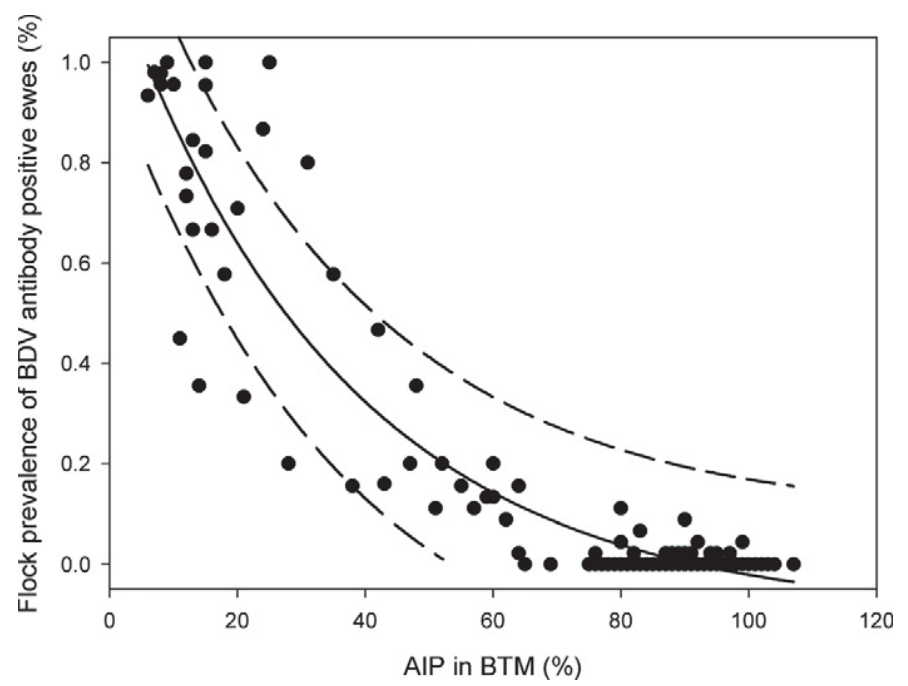

Figure 1. Observed within-flock prevalence (O) of border disease virus (BDV), predicted within-flock prevalence (solid line), and 95\% prediction interval (between dashed lines), according to different antibody inhibition percentage (AIP) values of 161 bulk-tank milk (BTM) samples. 
Table 1. Within-flock seroprevalence of border disease virus (BDV) in 161 dairy sheep flocks according to the antibody inhibition percentage (AIP) in bulk-tank milk (BTM) samples

\begin{tabular}{|c|c|c|c|c|}
\hline \multirow{2}{*}{$\begin{array}{l}\text { BDV AIP } \\
\text { in BTM }(\%)\end{array}$} & \multicolumn{3}{|c|}{ Within-flock prevalence (\%) } & \multirow[b]{2}{*}{ Total } \\
\hline & $<10$ & $10-30$ & $>30$ & \\
\hline$>65$ & 120 & 1 & 0 & 121 \\
\hline $35-65$ & 2 & 11 & 3 & 16 \\
\hline$<35$ & 0 & 1 & 23 & 24 \\
\hline Total & 122 & 13 & 26 & 161 \\
\hline
\end{tabular}

$$
\text { Prev }(\%)=129 \exp (-0.0279 \text { AIP })-1 .
$$

The predicted within-flock prevalence and the $95 \%$ prediction interval according to the AIP in BTM are shown in Figure 1.

In another approach, flocks were assigned to 3 categories according to their AIP in BTM (group A $>65 \%$, group $\mathrm{B} \leq 65 \%$ and $\geq 35 \%$, group $\mathrm{C}<35 \%$ ). The expected within-flock prevalence of BDV antibodypositive ewes was $<10 \%$ for group A, between 10 and $30 \%$ for group B, and $>30 \%$ for group C. In the present sample, only 7 flocks (4.35\%) had an observed seroprevalence outside the expected range (Table 1).

Because very few flocks had seroprevalence levels between 10 and 30\%, the analysis was focused on the ability of the blocking ELISA applied to BTM samples to distinguish low-seroprevalence flocks (i.e., prevalence $<10 \%$ ) from all others (i.e., prevalence $\geq 10 \%$ ). Based on efficiency and Youden's index estimates, the best decision threshold for AIP in BTM was $65 \%$, for which $\mathrm{Se}=97.4 \%(95 \%$ CI: 86.5-99.9\%) and $\mathrm{Sp}=97.5 \%$ (95\% CI: 93.0-99.5\%; Table 2). A cut-off value of $80 \%$ (that currently used in cattle) was associated with perfect sensitivity (Se $=100.0 \%, 95 \%$ CI: 91.0-100.0\%) but lower specificity ( $\mathrm{Sp}=85.2,95 \% \mathrm{CI}: 77.7-91.0 \%)$. For a cut-off value of $75 \%$, the test characteristics were intermediate. As expected, the test characteristic estimates based on Monte Carlo simulations were slightly lower than those based on crude estimates of seroprevalence (Table 2), but were still high. The $65 \%$ threshold value for AIP in BTM was associated with the best test efficiency and highest Youden's index, whereas the $80 \%$ cut-off value was associated with lower specificity.

Because false-negative results could be expected due to the non-perfect sensitivity of the blocking antibody ELISA applied to BTM, the evolution of the withinflock prevalence of antibody-positive ewes and of AIP in BTM was followed in the subsequent years using the same sampling scheme (i.e., 45 ewes tested per flock). Unfortunately, only 60 of the 121 flocks with initial AIP values $>65 \%$ could be resampled. Among them, $38(63 \%)$ could be monitored during the 2 subsequent milking campaigns. In 2 flocks, only 1 of 45 sampled ewes was antibody-positive (within-flock prevalence $2.2 \%, 95 \%$ CI: $0.0-11.8 \%$ ) and in another flock, 2 ewes yielded positive results (within-flock prevalence $4.4 \%$, 95\% CI: $0.5-15.1 \%$ ). Based on the absence of a strong increase in the prevalence of antibody-positive ewes and health problems, we were confident that none of the resampled flocks had undergone an active circulation of BDV during the 2 or $3 \mathrm{yr}$ of the monitoring period. All flocks had AIP values in BTM $>65 \%$ in the subsequent years, with 13 flocks $(21.7 \%$ ) having values between 65 and $80 \%$. This finding strengthens the idea that the $80 \%$ cut-off value suffers some lack of specificity for the detection of BDV-positive flocks.

Similarly, in 19 of the 41 flocks with initial BTM values $<65 \%$, representative samples of 45 milking ewes and BTM samples were tested for the presence of BDV antibody during $1(\mathrm{n}=11)$ or $2(\mathrm{n}=8)$ subsequent milking campaigns. The number of antibody-positive ewes ranged between 5 and 45 , suggesting no strong decrease in seroprevalence in these flocks. For all flocks except one, the AIP value in BTM was $<65 \%$ in the subsequent years. The unrecovered flock had a BTM value of $67 \%$, with 5 antibody-positive ewes in the 45 tested.

Our results are in agreement with those of Berriatua et al. (2006) and Garcia-Pérez et al. (2010) and show that BTM sample analysis can provide inexpensive and valuable information on flock exposure to pestiviruses

Table 2. Performance (and 95\% CI) of the blocking ELISA for border disease virus (BDV) applied to bulk-tank milk (BTM) samples according to different antibody inhibition percentage (AIP) threshold values

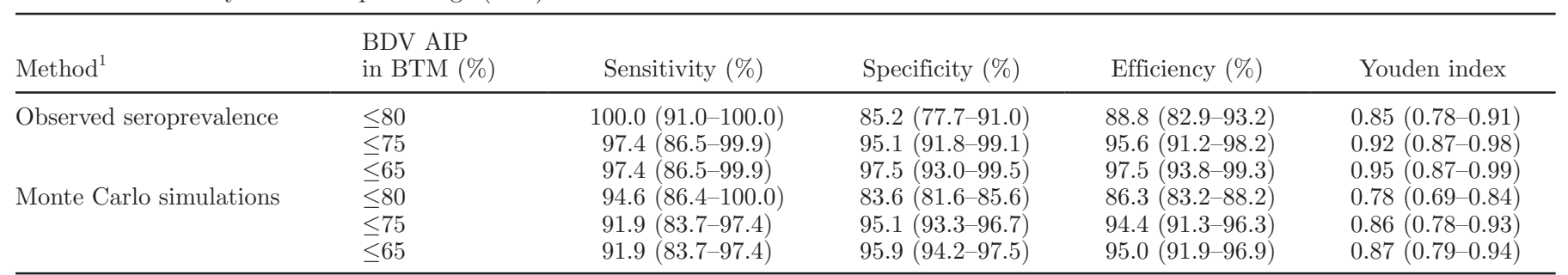

${ }^{1}$ Estimates were computed based on observed seroprevalence or by using Monte Carlo simulations. Flocks with seroprevalence $\geq 10 \%$ were considered positive. 
in dairy sheep. The high performance estimates of the blocking ELISA used allow for year-to-year monitoring of disease-free flocks in BDV control schemes. However, our findings suggest that the decision threshold value used in dairy cows herds to discriminate between lowand high-seroprevalence flocks is not adequate in sheep flocks.

\section{ACKNOWLEDGMENTS}

The authors thank all farmers and Pierre Blancard (Cabinet vétérinaire, Saint Afrique, France) for involvement in and dedication to this study. This study was funded by the Fédération des Organismes de Défense Sanitaire de l'Aveyron (FODSA, Toulouse cedex, France).

\section{REFERENCES}

Beaudeau, F., C. Fourichon, A. Robert, A. Joly, and H. Seegers. 2005. Bulk milk somatic cell counts and bovine viral diarrhoea virus (BVDV) infection in 7252 dairy herds in Brittany (western France). Prev. Vet. Med. 72:163-167.

Berriatua, E., J. F. Barandika, G. Aduriz, A. Hurtado, L. Estevez, R. Atxaerandio, and A. L. Garcia-Perez. 2006. Flock-prevalence of border disease virus infection in Basque dairy sheep estimated by bulk-tank milk analysis. Vet. Microbiol. 118:37-46.

Dubois, E., P. Russo, M. Prigent, and R. Thiery. 2008. Genetic characterization of ovine pestiviruses isolated in France, between 1985 and 2006. Vet. Microbiol. 130:69-79.

Garcia-Pérez, A. L., F. Ruiz-Fons, J. F. Barandika, G. Aduriz, R. A. Juste, and A. Hurtado. 2010. Border disease virus seroprevalence correlates to antibodies in bulk-tank milk and reproductive performance of dairy sheep flocks. J. Dairy Sci. 93:2444-2449.

Houe, H., A. Lindberg, and V. Moennig. 2006. Test strategies in bovine viral diarrhea virus control and eradication campaigns in $\mathrm{Eu}-$ rope. J. Vet. Diagn. Invest. 18:427-436.

Lindberg, A., J. Brownlie, G. J. Gunn, H. Houe, V. Moennig, H. W. Saatkamp, T. Sandvik, and P. S. Valle. 2006. The control of bovine viral diarrhoea virus in Europe: Today and in the future. Rev. Sci. Tech. 25:961-979.

Messam, L. L. McV., A. J. Branscum, M. T. Collins, and I. A. Gardner. 2008. Frequentist and Bayesian approaches to prevalence estimation using examples from Johne's disease. Anim. Health Res. Rev. 9:1-23.

Nettleton, P. F., J. A. Gilray, P. Russo, and E. Dlissi. 1998. Border disease of sheep and goats. Vet. Res. 29:327-340.

Schisterman, E. F., and N. Perkins. 2007. Confidence intervals for the Youden index and corresponding optimal cut-point. Comm. Statist. Simulation Comput. 36:549-563.

Valdazo-González, B., M. Alvarez-Martinez, and T. Sandvik. 2007. Genetic and antigenic typing of border disease virus isolates in sheep from the Iberian Peninsula. Vet. J. 174:316-324.

Valle, P. S., S. Wayne Martin, and E. Skjerve. 2001. A Bayesian approach to estimating the performance of a bovine virus diarrhoea virus (BVDV) antibody ELISA bulk-tank milk test. Prev. Vet. Med. 50:71-87. 